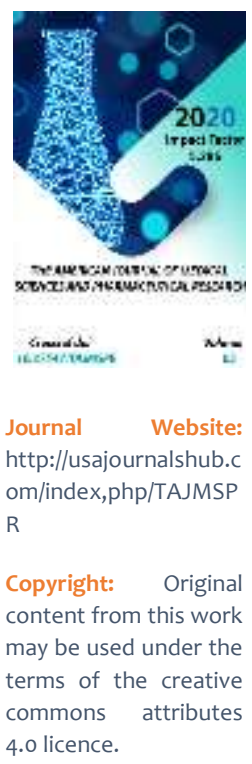

\title{
Results Of Examination Of Patients With Radiculopathies In Chronic Brucellosis
}

Sokhiba Khakimova Ziyadullayevna, Course Of Neurology And Neurosurgery Postgraduate Faculty Samarkand Medical Institute, Samarkand, Uzbekistan

Dildora Atokhodjaeva Alisherovna

Teaching Assistant In Department Of General Professional And Special Disciplines, Faculty of Tourism Management"Silk Road" International University Of Tourism Samarkand, Uzbekistan

\section{ABSTRACT}

The article presents the features of the clinical course of chronic brucellosis at the present stage, the issues of diagnostics and treatment, taking into account the results of our own observations of patients with damage of the nervous system and musculoskeletal system. There is characteristic of clinical and epidemiological, laboratory and instrumental diagnostics of chronic brucellosis, the issues of etiopathogenetic therapy are considered.

\section{KEYWORDS}

Brucellosis, diagnostics, treatment, Milgamma.

\section{INTRODUCTION}

The steady increase of the brucellosis incidence is becoming a global problem in the world. Brucellosis is a particularly dangerous and socially significant infection, that causes significant economic damage and a high level of disability of patients. Disorders from the nervous system in chronic brucellosis (CB) are one of the most common, and lesions of both the central nervous system are detected, the clinic of which fits into the syndromes of arachnoiditis, meningoencephalitis, myelitis, and the peripheral department in the form of polyneuropathies, radiculopathies, plexitis, neuritis $[1,3,6]$. 
At the present stage of treatment of patients, three directions are distinguished: specific treatment with brucellin, pathogenetic and symptomatic therapy [4]. Currently, the most attention is paid to the issues of rehabilitation treatment, which is the most promising direction in the treatment of patients with peripheral form of chronic brucellosis [7,8].

The aim of the study is to improve the pain treatment with damage of the nervous system of patients with brucellosis $[1,2,4]$.

\section{MATERIALS AND RESEARCH METHODS}

Under our supervision, there were 32 patients complaining of pain, which are: women - $85 \%$, men $-15 \%$. The age division is: from 24 to 30 years - $11.5 \%$; from 30 to 40 years old - $19.2 \%$; from 40 to 50 years old - $15.4 \%$; from 50 to 60 years old $-38.5 \%$; older than 60 years $-15.4 \%$, the age of patients - from 39 to 84 years $(53.7 \pm$ 2.3). The duration of the disease ranged from 0 to 52 years: $51.6 \%$, patients were ill for up to 5 years, $19.4 \%$ of patients $-5-10$ years, $10-15$ years $-9.6 \%, 19.4 \%$.

All patients were divided into two groups: the main - 24 patients who received traditional treatment with the addition of Milgamma and the control group - who received only traditional treatment.

Clinical and epidemiological examination with specific laboratory diagnostics (enzyme-linked immunosorbent assay with antibodies to brucellosis pathogens (Br. Abortus, $\mathrm{Br}$. Melitensis), Haddlson plate agglutination test, Rites agglutination test in test tubes, indirect hemagglutination reaction with brucellosis diagnostic, Coombs antiglobulin reaction, Burne skin-allergic test). From additional methods, clinical, biochemical and instrumental (radiography of the joints and spine) studies were carried out. The dynamics of pain perception was evaluated on a 10-point visual analogue scale (VASh) before and after drug administration.

\section{RESULTS AND DISCUSSION}

In the primary epidemiological diagnosis of brucellosis in most patients (61.5\%), the professional nature of the disease with a contact path of infection was established: $38.5 \%$ were workers at the meat processing plant who received specific prophylaxis irregularly, with long interruptions, and also did not always use personal protective equipment ( gloves), often noted scratches, cuts, bruises in the processing of raw meat. Veterinarians accounted for $7.6 \%$, who are infected by contact with sick animals (cattle, pigs). $11.4 \%$ were milkmaids, possibly infected while caring for cattle, and were also in contact with sick animals, their excreta. In $3.7 \%$ of the patients, a presumably mixed (aerogenic and alimentary) route of infection was observed (they were engaged in shearing sheep, where there were cases of brucellosis among animals, as well as raw milk and dairy products were consumed). In $37.5 \%$ of patients, an alimentary route of infection was observed: eating unboiled milk and raw dairy products: feta cheese, cream, sour cream, etc.

As a result of a clinical examination, taking into account the timing of infection and the epidemiological history, we found chronic brucellosis with a combined lesion of the osteoarticular and nervous systems in $65.3 \%$ of patients, and a primary lesion of the osteoarticular system in 35.6\%. The changes are identified during radiography of the joints, which indicating a deeply advanced process: deforming arthrosis of the knee joints - in 46.1\%; deforming arthrosis of the hip joints - in 33.6\%; humeroscapular periarthrosis - in $27.7 \%$; deforming arthrosis of the wrist joints and joints of the hands - in 30.5\%; deforming 
arthrosis of the interphalangeal and metatarsophalangeal joints of the feet - in 11.5\%; degenerative-dystrophic changes in the spine (osteochondrosis, subchondral osteosclerosis, deforming spondylosis, uncovertebral arthrosis) - in $80.2 \%$ of patients

(Fig. 1).

\section{НазваChanges identified during joint radiographyние диаграммы}

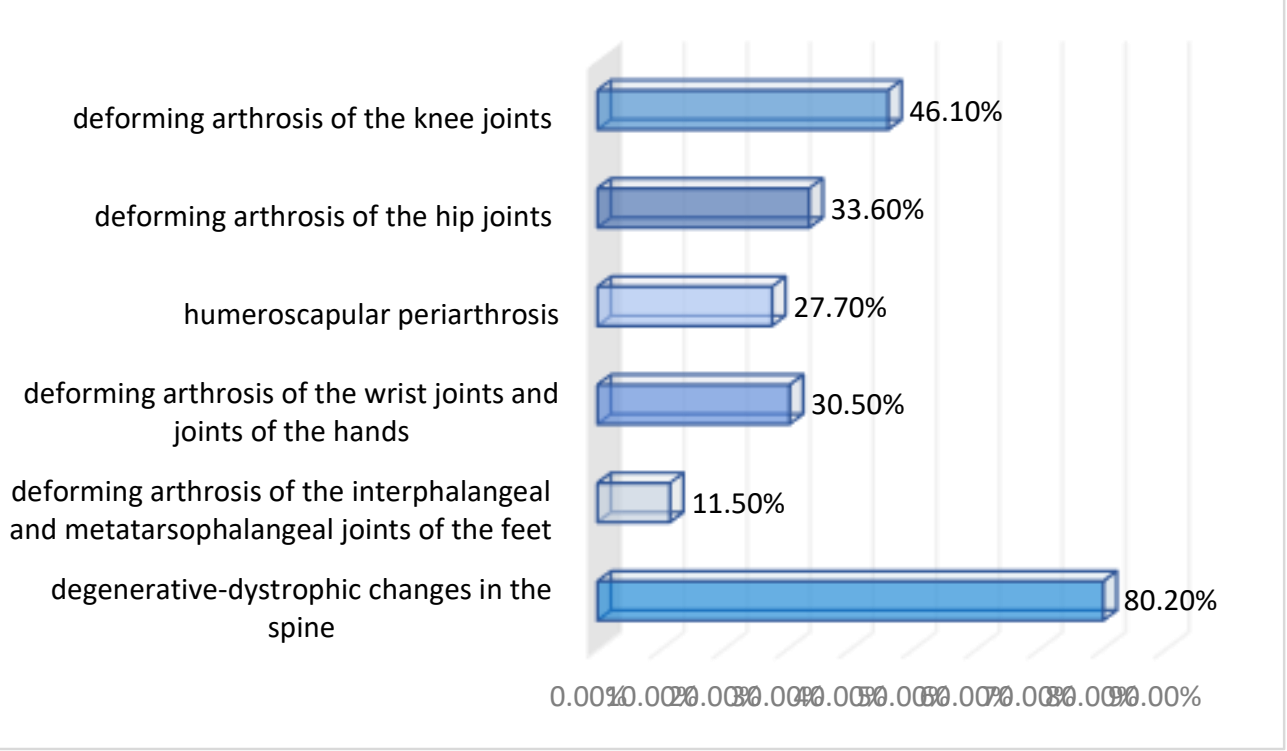

Fig. 1. Changes identified during joint radiography.

The most common and characteristic for brucellosis is the defeat of the sacroiliac joints sacroileitis, which is rare in rheumatism and other infectious diseases, which allows the patient to suspect a brucellosis process [7]. Changes in the sacroiliac joints rarely attract the attention of practitioners in connection with the presence of concomitant symptoms from the muscles, sacral and sciatic nerves, therefore, sciatic nerve neuritis or osteochondrosis of the lumbosacral spine with radicular syndrome is more often diagnosed.

Lesions of the soft skeleton in most cases manifest as inflammatory nodules in the connective tissue, the so-called fibrositis, pathognomonic for brucellosis, especially for primary chronic forms. They can form in the form of seals of various sizes and soreness everywhere, where there is connective tissue: in subcutaneous fat, fascia, aponeurosis, periosteum, surrounded by joints, nerve trunks and roots, they may later dissolve or remain in the form of scars $[1,4,9]$.

The development of the clinic of modern brucellosis was reflected in the features of damage of the nervous system. Lesions of the peripheral nervous system became softer, and the central nervous system became rarer or not observed at all $[1,10]$. So, in chronic brucellosis (8.7\%), neurological syndromes often occur with a variety of diffuse symptoms, 
complex types of disorders of sensitivity and motor sphere (for example, the type of brain tumor, multiple sclerosis, stem encephalitis, etc.); the most common symptoms of a functional nature in the form of neuralgia (35.6\%) [4]. In patients with chronic brucellosis observed by us, lumbar ischalgia, neurodystrophic form, cervicalgia, sensorimotor polyneuropathies and other disorders were recorded in $30.5 \%$ of cases.

The clinical and epidemiological diagnosis was confirmed by the results of specific laboratory diagnostics: by IFA in $46.2 \%$ of patients, antibodies to the causative agents of brucellosis (Br. Abortus, Br. Melitensis) of the IgG and IgM classes were determined, in 57.7\% - by the positive Hedlson agglutination test. In $46.1 \%$ of patients, a positive Rite agglutination reaction was determined in the titer from $1 / 200$ to $1 / 800$; as well as in $34.6 \%$ of patients, positive results were simultaneously observed both in IFA and in the agglutination reaction of Rite and Hedlson. It is important that the Byurne allergic skin test in all patients (100\%) with chronic brucellosis was positive.

The revealed "spread" of the frequency of positive serological reactions in chronic brucellosis is natural and indicates the need to use at least two methods. The coincidence of positive IFA results with Rite agglutination reaction was found in $46.0 \%$ of cases, with indirect hemagglutination reaction - in $37.0 \%$; with the Coombs antiglobulin reaction - in 100\% $[4,11]$. The Burne test refers to tests of the third level of diagnosis to detect the sensitization of the body to brucella, its degree reflects the final stage of the interaction of the antigen and the immune system [9].

The tactics of treating patients with chronic brucellosis is determined by the phase and form of the disease and the state of the reactivity of the body, which confirmed by the results of clinical, laboratory and instrumental examination. The issue of prescribing antibacterial therapy, which is not indicated for chronic inactive and residual brucellosis, as well as for the prevention of relapse, remains controversial in real practice. Indications for the appointment of antibiotic therapy are not sufficient only positive serological tests and allergy tests, and most importantly - a clinical exacerbation of chronic brucellosis infection. We used etiotropic therapy for the treatment of patients with chronic brucellosis during exacerbations: tetracycline antibiotics (iv videoccin or doxycycline per os) - in $77.9 \%$; aminoglycosides (gentamicin) - in 56.7\%; III generation cephalosporins (ceftriaxone) - in $23.7 \%$ of cases.

But still, the leading principle in the treatment of chronic brucellosis is pathogenetic using non-steroidal anti-inflammatory drugs (diclofenac, ortofen, etc.), given the persistence of the inflammatory process, desensitizing agents (mainly groups of mast cell membrane stabilizers - ketotifen, azelastine, lodoxamide, etc.) as well as metabolic correction preparations (B vitamins, almiba, nicotinic acid, pentoxifylline, cytoflavin, reamberin, remaxol, etc.), taking into account oxidation-reduction disorders that correlate with the degree of adjustment allergic organism $[2,4,11]$.

During a recurring course of the disease, as well as with a chronic process, immunotropic therapy is indicated, taking into account the degree of immunological rearrangement of the patient's body with brucellosis. The appointment of immunocorrective therapy is considered necessary 1-3 weeks after taking antibiotics. It is recommended to use immunocorrectors of various groups: immunomodulators of endogenous origin (immunoregulatory peptides obtained from 
central organs of the immune system (thymus and bone marrow), cytokines, interferons, immunomodulators of exogenous origin (pyrogenal, zymosan, prodiogisan, lycopid, bacterial lysates), synthetic immunomodulators (levamisole, pentoxyl, methyluracil, immunofan), cycloferon, an interferon inducer $[11,12]$.

Patients were divided into 2 groups: in the main group (22 patients), milgamma was prescribed in combination with the above traditional treatment, in the control group (10 patients) only traditional therapy was performed. The effectiveness of treatment was evaluated on the 10th day and 24th day on the visual analogue scale, as well as after 3 and 6 months (Fig. 2). Throughout the study, according to the visual analogue scale, there was a tendency to a higher efficiency of the milgamma combination in relation to traditional treatment, however, this difference reached the level of statistical significance only by the 24th day. Using the neuropathic pain scale, it was shown that only against the background of combination therapy, such characteristics of pain as intensity and severity significantly decreased, pain tolerance improved. A significant and moderate effect against the background of the introduction of milgamma was observed in $66 \%$ of patients, in the control group - in 34\% ( $p$ <0.05). 3 months later pain was absent or was minimal in $63 \%$ of patients in the main group and in $50 \%$ of patients in the control group ( $p<0.05)$. The results obtained indicate that the complex of $B$ vitamins can potentiate the analgesic effect of non-steroidal anti-inflammatory drugs and contribute to a more rapid and persistent regression of pain.

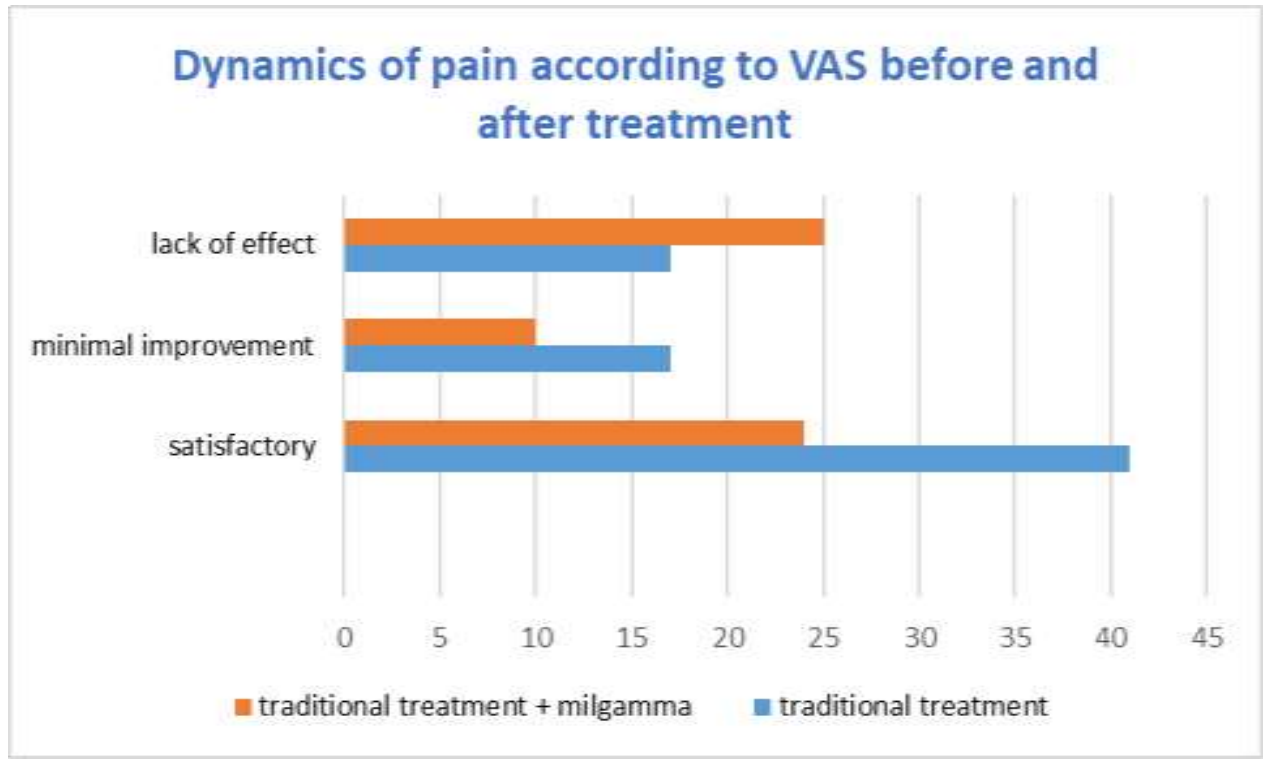

Fig. 2. Dynamics of pain according to VAS before and after treatment

According to the general clinical impression scale against the background of the use of combination therapy, including milgamum, on the 10th day a significant effect was noted in 6 patients $(25 \%$ of the patients who entered the study), satisfactory (moderate - in $19(41 \%)$, minimal improvement - 8 (17\%), no improvement - 7 (17\%). In the control group, a significant effect was observed in 2 patients (10\%), satisfactory - in $4(24 \%)$, minimal 
improvement - in 9 (41\%), lack of effect - in 5 (25\%). The effectiveness of therapy, initial intensity and length flaccidity, the presence of symptoms of loss, the severity of vertebral syndrome were not affected by the age.

\section{CONCLUSIONS}

Thus, the diagnosis and treatment of chronic brucellosis in the practice of a neurologist is a difficult task and is determined by the incidence rate in the region, both among animals and among people, the professional readiness of practical doctors and the availability of diagnostic technologies. At the same time, it should be taken into account the epidemiological history, the predominance of latent and primary chronic forms of low activity with damage to the joints and nervous system (with characteristic polyofocal), autonomic reactions, the presence of fibrositis, prolonged subfebrile condition, which determine the clinical differential diagnosis. Specific diagnostics are determined by various methodological levels - from the isolation of the pathogen to the determination of its antigens and antibodies, as well as the degree of sensitization to brucella. Hence, the treatment of chronic brucellosis depends on the activity of the infectious process, which requires the appointment of antibacterial agents and reasonable immunotropic therapy.

The received results confirm the efficacy and safety of milgamma preparations in the treatment of pain in patients with damage to the nervous system in brucellosis. They suggest that high doses of neurotropic vitamins as part of milgamma preparations can potentiate the analgesic effect and contribute to a faster regression of the pain syndrome. The peculiarity of our study was that, firstly, we showed the effectiveness of a complex of $B$ vitamins (using the example of a milgamma preparation) with such a severe form of back pain as vertebrogenic radiculopathy. Secondly, we found that milgamma effectively acts on the neuropathic component of brucellosis pain. Thirdly, it is shown that the addition of a 14-day course of oral administration of the drug milgamma compositum to the 10-day course of intramuscular administration of milgamma promotes a more complete manifestation of the therapeutic potential of the drugs. Finally, the data obtained indicate that the effectiveness of the combined course of treatment with milgamma can be observed not only in the short, but also in the medium term (3 months), which was also facilitated by its prolongation by oral administration of the milgam compositum tablets.

\section{REFERENCE}

1. Belozerov E.S. Brucellosis. - Leningrad: Medicine, 1985. - $183 \mathrm{p}$.

2. Infectious diseases: national leadership / Ed. N.D. Yushchuk, Yu.Ya. Vengerova. - M.: GEOTAR-Media, 2009 .-- S. 396-406.

3. Doganay, M., \& Aygen, B. (2003). Human brucellosis: an overview. International journal of infectious diseases, 7(3), 173182.

4. Dahouk, Sascha \& Tomaso, Herbert \& Nöckler, Karsten \& Neubauer, Heinrich \& Frangoulidis, Dimitrios. (2003). Laboratory-based diagnosis of brucellosis-a review of the literature. Part II: serological tests for brucellosis. Clinical laboratory. 49. 577-89.

5. Ariza $J$ et al. 2007. Perspectives for the Treatment of Brucellosis in the 21st Century: The Ioannina Recommendations. PLoS Med. 4(12): e317.

6. Prevention and laboratory diagnosis of brucellosis in humans: Guidelines MU

7. 1189 - 03. - Moscow: Federal Center for Sanitary and Epidemiological Supervision 
of the Ministry of Health of Russia, 2003. $58 \mathrm{p}$.

8. Kurmanova K.B., Duseynova A.K. Brucellosis. Clinical aspects. - Almaty: Kitap, 2002 .-- 352 p.

9. Kasatkina I. L., Beklemishev N. D. The pathogenesis of joint damage in brucellosis - Alma-Ata, 1976. - 232 p.

10. Knateeb M.J. Brucella arthritis: a study of 96 cases in Kuwait // Ann.Rheum.Dis. 1990 - V. 15. - P. 994-998.

11. Nasonov E.L., Bunchuk N.V. Rheumatic diseases. - M .: Medicine, 1997 .- 520 p.

12. Brucellosis / Ed. P.A. Vershilova. - M.: Medicine, 1972.- 436 p.

13. Beklemishev N.D. Chronic and latent brucellosis. - Alma-Ata: The science, 1965 -$-332 \mathrm{p}$.

14. Bryzhakhin G.G., Belozerov E.S. Brucellosis (damage to the nervous system). - Almaty, 1993 -- 158 p.

15. Kurmanova G.M., Duseynova A.K., Kurmanova K.B., Spiricheva N.Kh. Evaluation of the immune status and differential immunocorrection for brucellosis: Guidelines. - Almaty, 2002 .-- 30 s.

16. Brucellosis. Modern approaches to therapy: A manual for doctors / Edited by A.A. Shuldyakova, M.G. Romantsova.Saratov- St. Petersburg, $2006 .--28$ p.

17. Khakimova S.Z., Jurabekova A.T. 2016, Clinical diagnosis and treatment of chronic brucellosis in real practice of a neurologist. Medicine. Alma Ata No. 7, (169) 2016, p. 68-72

18. Nasretdinova M. T., Karabaev H. E. Vestibular neuronitis-the problem of systemic dizziness //European science review. - 2019. - T. 2. - №. 1-2.

19. Al-Tawfiq JA. 2008. Therapeutic options for human brucellosis. Expert Rev Anti Infect Ther. 6(1): 109-120.
20. Solera J. 2010. Update on brucellosis: therapeutic challenges. Intl J Antimicrob Agent. 36S, S18-S20. 